IV Congreso Internacional Estética y Política Poéticas del desacuerdo para una democracia plural 16 y 17 de octubre. Valencia

Doi: http://dx.doi.org/10.4995/CEP4.2019.10506

\title{
Materialismo dialéctico y política. Reformulación de Slavoj Žižek
}

\section{Eduardo Abril Acero}

UNED. abril_eduardo@hotmail.com

\begin{abstract}
Slavoj Žižek is not one of the most prolific authors of recent decades. His political proposal is far from the usual positions of the left, which focus on the defense of the logic of diversity. His bet is a recovery of a strong dialectical materialism, based on his Lacanian reading of Hegel. This text analyzes the theoretical consistency of his dialectical materialism and attempts to answer the question "what policy should a dialectical materialist defend today?" From the author's point of view. Žižek defends a materialism that supports political action. That is why it separates itself from Darwinist materialism, orthodox Marxist materialism and multicultural materialism. His intention is to think of a new critical position exemplified by the dialectical relationship between Melville's Bartleby and his notion of self-coup.
\end{abstract}

Keywords: Dialectical materialism, Absolute Knowledge, Hegel, self-coup, Bartleby

\begin{abstract}
Resumen
Uno de los autores más prolíficos de las últimas décadas es sin duda Slavoj Žižek. Su propuesta política, se aleja de las posturas habituales de la izquierda, centradas en la defensa de lógicas de la diversidad, y propone la recuperación de un materialismo dialéctico fuerte, fundamentado en su lectura lacaniana de Hegel. En este texto se analiza la consistencia teórica de la versión del materialismo dialéctico propuesto por Žižek con la intención de responder la pregunta: “¿qué política debería defender hoy en día un materialista dialéctico?”. Žižek defiende un materialismo que fundamente la acción política. Por eso se desvincula del materialismo darwinista, del materialismo marxista ortodoxo y del materialismo multicultural. Su intención es pensar una nueva posición crítica ejemplificada por la relación dialéctica que mantiene el Bartleby de Melville con su noción de autogolpe.
\end{abstract}

Palabras clave: Materialismo dialéctico, Saber Absoluto, Hegel, Autogolpe, Bartleby. 


\section{Introducción.}

En dos de sus últimas obras, Menos que nada y Contragolpe Absoluto, Žižek se centra en un replanteamiento completo del materialismo dialéctico. Esta propuesta debe ser entendida como parte de su proyecto político de repetir Lenin, que no equivale a una reedición o adaptación del marxismo-leninismo a los nuevos tiempos, pues en el siglo actual se presenta ya como una vía muerta para la filosofía y la acción política. Repetir Lenin, en el sentido que lo piensa Žižek, igual que repetir el materialismo dialéctico, pasa por un replanteamiento completo de las posiciones del postmarxismo. No se trata, como ha señalado Agon Hamza, de reparar la vía herida del marxismo contemporáneo después del desastre soviético, sino de repetir por completo el proyecto revolucionario marxista: "Comenzar desde el principio no significa volver a donde nos detuvimos, es decir, a los proyectos socialistas del siglo XX en todas sus variedades, sino volver a su punto de partida y comenzar de nuevo" (Hamza, 2016). Para tal repetición Žižek considera que la vuelta al marxismo pasa por una recuperación de Hegel, pero no el Hegel de la tradición idealista, sino un Hegel materialista. Por eso, la repetición del materialismo dialéctico no consiste solamente en desembarazarse del materialismo vulgar del marxismo-leninismo que, a día de hoy, se ha convertido en una teoría desproblematizadora inoperante, sino más bien y sobretodo, en reconstruir desde cero la dialéctica materialista a la luz de una nueva revisión del cruce entre Marx y Hegel. Tampoco es un mero replanteamiento teórico, tratando de sacar a la luz líneas de investigación que han quedado cegadas en la tradición del hegelianismo y el marxismo, sino que el objetivo es abrir la posibilidad de una nueva forma de acción política emancipadora, pues el objetivo último de la reflexión Zizekiana va en la dirección de rescatar la posibilidad de seguir siendo materialistas dialécticos en el siglo XXI.

\section{Crítica del materialismo contemporáneo.}

Žižek pone la misma objeción a las formas del materialismo contemporáneo que pueden reducirse a grandes rasgos a tres: el materialismo dialéctico marxista-leninista ortodoxo, el materialismo darwinista, y lo que llama "materialismo democrático" o también "materialismo deleuziano", encarnado especialmente en el concepto de materia vibrante de Jane Bennet (Bennet, 2010). El argumento para rechazarlos todos, aunque adopta diferentes formas en los textos de Žižek, se puede cifrar en un solo punto. Todas estas formas de materialismo esconden la referencia una posición exterior que les hace caer inevitablemente en un idealismo teológico velado. Lo que se debe desterrar del materialismo contemporáneo, por tanto, es la tesis de que la realidad está sin más "ahí fuera". Este modo de pensamiento no evita la ontología premoderna que considera la realidad como una sustancia completa y acabada, aún cuando se conciba como un proceso dinámico en constante cambio. Žižek da cuenta del error que se comete desde este argumento: incluso cuando tratan de ser explicaciones de la totalidad evitan incluir la propia posición dentro de esta misma explicación. Por eso, el verdadero materialismo, para Žižek, pasa por reconocer la tesis hegeliana de que "no es que hay una realidad fuera de nuestra mente, sino que no hay una mente fuera de la realidad" (Žižek, 2006), lo que coincide con el Saber Absoluto hegeliano, aunque redefinido por Žižek como la posición del reconocimiento de que la realidad toda, en sí misma, está ya de salida no totalizada. Por eso, señala Castro Gómez en su lectura de Žižek, que "la postura materialista correcta no es que el universo sea material, sino que es ontológicamente incompleto" (Castro Gómez, 2015).

Es aquí donde encontramos la verdadera diferencia entre materialismo e idealismo: el idealismo es un modo de pensar lo real que apela siempre a una posición exterior, mientras que el materialismo, más allá de ser un mero corporeísmo, consiste en la aceptación de una realidad única. Pese a que la filosofía materialista haya pretendido centrar su análisis en la consideración de que todo lo real se reduce a la materia y sus principios, el problema es que cuando se encuentra con las contradicciones e inconsistencias propias de todo sistema, se traiciona a sí misma y postula veladamente un afuera que explique estas turbulencias internas. Esta filosofía procede de acuerdo con lo que Lacan llamaba la lógica masculina, la lógica del todo y la parte. Por ejemplo, el materialismo dialéctico del marxismo-leninismo fundamenta su análisis en el reconocimiento de las contradicciones intrínsecas a toda realidad social, contradicciones que deben ser resueltas en la dirección de una sociedad reconciliada. Aunque parecen aceptar una visión problematizadora de lo real, puesto que toman en cuenta los antagonismos sociales, también suponen que tales contradicciones y desequilibrios sociales son algo que se resuelve a través la construcción de una sociedad más racional. De este modo, pese a que la realidad es tomada como un 
turbulento torbellino de antagonismos y enfrentamientos, un hegeliano banco de matadero, esta visión es completada con lo que Lacan llamaba "la perspectiva del juicio final" (Lacan, 2007). Se trata de la idea de que la realidad y la historia avanzan inexorablemente, a través de sus contradicciones internas, hacia un aumento de la racionalidad. A esta idea Lacan se refiere como la fantasía de la segunda muerte: la muerte "a la cual se puede aún apuntar cuando la muerte ya ha sido lograda" (Lacan, 2007). En el seminario VII, el psicoanalista francés, vincula el mantenimiento del deseo con la adopción por parte del sujeto de una posición absoluta bajo la forma de una fantasía. No se trata de que un materialista dialectico clásico, un demócrata liberal o un multiculturalista adopten explícitamente una posición absoluta y dicten desde ahí la acción política. Aún cuando revelásemos la inoperancia o la impostura de un materialista dialéctico o de un liberal multiculturalista, su fantasía se mantiene, pues su posición se fundamenta en el núcleo mismo del deseo: el deseo quiere su realización, demanda un final donde su anhelo sea satisfecho, y esto equivale a adoptar la posición del propio final, de la propia muerte, postulando una «segunda muerte» fantasmática en la que todos los anhelos son colmados. Aquí estaría, según el análisis de Žižek el error del materialismo, ya adelantado por el propio Lacan: sigue consistiendo en una posición idealista puesto que el sujeto para adoptar la perspectiva del Juicio Final debe tacharse a sí mismo de la ecuación, es decir, debe postularse a sí mismo como un elemento fuera del conjunto de lo real desde la propia muerte subjetiva.

\section{El nuevo materialismo dialéctico de Žižek.}

Contra este idealismo velado, Žižek recupera la idea paulina de que el tiempo final ya ha llegado y debemos estar preparados. Esto equivale a la posición final de la fenomenología, el saber absoluto reinterpretado como la verdadera posición materialista, en el sentido de superar completamente cualquier dualismo o pluralismo ontológico, y no postular ningún afuera. La filosofía materialista debe hacer completamente la experiencia real de la muerte de dios sin llenar el vacío que queda mediante un nuevo objeto de deseo.

Esta es la razón por la que debemos comprender en qué consiste el replanteamiento que hace Žižek de la dialéctica y el Saber Absoluto hegeliano. A grandes rasgos podemos decir que, mientras que la tradición marxista-leninista pone el énfasis en la construcción de totalidades y por eso concibe la dialéctica como un proceso que avanza, a través de sus contradicciones, hacia una sociedad racionalizada, Žižek piensa a Hegel más como "un pensador de rupturas decisivas, de contradicciones irreconciliables, de pérdidas que nunca se recuperan" (Kotsko, 2008), acentuando el elemento triturador de la negatividad hegeliana, que hace coincidir con la pulsión de muerte freudiana y piensa la dialéctica no como un proceso de construcción, sino como un método que nos permite hacer un cambio de paralaje, donde lo que inicialmente se presenta como un obstáculo, es pensado más adelante como el elemento que permite sostener mínimamente una posición dada. Dicho de otro modo, la dialéctica no es para Žižek, como ha señalado Carla Cordua, el "orden del progreso que supera las diferencias y antagonismos del mundo" (Cordua, 2013), sino más bien un método para insistir en una posición parcial con la esperanza de que ésta se universalice.

La historia no es un despliegue lineal de la idea, de forma que se va construyendo el futuro mediante una evolución o un progreso. Es la negatividad la que está detrás del sucederse de los acontecimientos, la negación y aniquilación de toda estructura que pretende ser estable: "el llamado 'desarrollo dialéctico' consiste en la incesante repetición de un comienzo exnihilo, en el aniquilamiento y la reestructuración retroactiva de los contenidos presupuestos" (Žižek, 2010). De este modo, esta negatividad, la imposibilidad de clausura ontológica actúa positivamente produciendo efectos reales en el campo de la realidad material. Precisamente por esto el materialismo que propone Žižek es dialéctico, dado que es la propia negatividad-inconsistencia engendrada por el campo del funcionamiento de lo material lo que actua como sostén y a la vez como motor de cambio de esta realidad.

Resulta central comprender, por tanto, qué significado tiene para el método dialéctico la «negación de la negación» hegeliana. Desde luego que no se trata ya de la superación de la contradicción mediante la integración de sus partes en una unidad superior, ahora verdaderamente reconciliada. La negación de la negación apunta más bien, para el filósofo esloveno, a un redoblamiento de la negación, un cambio de paralaje que hace aparecer aquello que se trataba de suprimir como la clave de bóveda de la nueva posición. Adam Kotsko lo ha expresado con claridad cuando afirma que "Se establece una posición o tesis, y luego se hace otra afirmación que, dentro del marco de la primera posición, aparece como una negación o antítesis. La síntesis no supera la negatividad de la antítesis, sino que la radicaliza hasta tal punto que ya no aparece como una negación" (Kotsko, 2008). Dicho de otro modo: lo que queda afirmado en la negación de la negación 
es la propia imposibilidad de la posición inicial, y es esta imposibilidad lo que se universaliza. Lo que se produce es el paso de la universalidad abstracta, la posición inicial presupuesta retroactivamente, a la universalidad concreta, por la que un elemento particular negativo ocupa el lugar de la universalidad. Žižek lo aclara decisivamente en sus dos últimas obras dedicadas al materialismo dialéctico, Menos que nada y Contragolpe absoluto: se trata de una posición que retroactivamente postula sus propias condiciones. Es decir, la universalidad abstracta en la que se incide tras su fracaso sólo existe en el proceso retroactivo de su postulación desde la universalidad concreta, es la propia pérdida, la imposibilidad de afirmarse como un «en sí», lo que se recupera como el elemento positivo que sostiene una realidad dada. La cuestión, por tanto, no está en subrayar las inconsistencias y fracasos de todo sistema en su aspiración de ser, sino de mostrar que es el propio fracaso el elemento que sostiene el orden. Por eso Žižek señala que "entramos a la modernidad cuando el fracaso ya no se concibe como opuesto al éxito, dado que el éxito mismo puede consistir únicamente en asumir heroicamente la plena dimensión del fracaso mismo, 'repitiendo' el fracaso como 'propio'” (Žižek, 2006). Es esta noción de repetición lo que hace de la realidad una realidad dialéctica, pues toda cosa no es sino la propia repetición de su fracaso positivizándose como tal.

Pero la dialéctica para Žižek no es sin más un mero método de exposición, un mapa de la construcción de las categorías. Suele entenderse la diferencia entre entendimiento y razón en Hegel apuntando a la idea de que, por un lado, el entendimiento es la facultad de romper, de aislar, de separar, reduciendo la diversidad de lo real a conceptos muertos, mientras que la Razón restablece esta diversidad sintetizando de nuevo los conceptos, dotándolos de una dinamicidad. Sin embargo, Žižek nos dice, que el paso del entendimiento a la razón no es este, sino el paso que damos cuando esta operación de separar y desgarrar la realidad que pertenece a nuestra mente como facultad, es transferido a la realidad misma y es la propia realidad la que se separa y se desgarra. "El pensamiento filosófico como tal comienza cuando somos conscientes de que ese proceso de "abstracción" es inherente a la realidad: la tensión entre la realidad empírica y sus determinaciones 'abstractas' conceptuales es inmanente a la realidad, es una característica de las 'cosas mismas"' (Žižek, 2015) o, dicho de otro modo, "las grietas y los vacíos de nuestro conocimiento de la realidad son simultáneamente las grietas y los vacíos del edificio ontológico 'real”" (Žižek, 2001).

Generalmente describimos el proceso de la comprensión dialéctica en Hegel del siguiente modo: tratamos de hacer la experiencia de la conciencia mediante la comprensión de un hecho, de acuerdo con nuestros esquemas conceptuales. Este proceso fracasa tarde o temprano y vemos que tales concepciones no son adecuadas para hacer esa experiencia del objeto, así que desechamos estas nociones como señales de su deficiencia y las sustituimos por nociones contrarias que tratan de salvar los errores de las nociones iniciales. Así ocurre una y otra vez en la Fenomenología. Pero sucede también que en este proceso nos darnos cuenta de algo inesperado: que las propias inconsistencias de nuestras nociones están inscritas en el objeto mismo. Y esto es lo que nos lleva a considerar que "la 'cosa misma' es inconsistente, que oscila entre sus diferentes determinaciones y es precisamente el despliegue de estas tensiones, esta lucha, lo que hace que esté "viva"” (Žižek, 2015). Caemos en la cuenta de que la noción de partida no está equivocada, que su dificultad no es una deficiencia de la propia noción, sino el hecho positivo que afecta a la realidad misma, así que esa deficiencia es postulada retroactivamente como inherente al propio mundo, el obstáculo inicialmente es transformado en el elemento que sostiene lo real. Aquí tenemos lo que podría ser un verdadero principio ontológico en Žižek: no es que las ideas sean un campo autónomo que en su desarrollo y en sus contradicciones a la hora de intentar comprender el mundo progresen, sino que , más bien, es lo real mismo lo que está aquejado de inconsistencia de modo paralelo a nuestra comprensión de la realidad: “cada nivel surge de la contradicción y está marcado por la contradicción” (Kotsko, 2008).

Si, a la luz de lo dicho, nos centramos en las cuestiones políticas, hacemos la pregunta de por qué no se sostienen y funcionan sin fricciones los sistemas políticos, las respuestas que encontramos desde el materialismo que Žižek quiere superar suelen ser del tipo: tal vez aun la ciencia no ha desarrollado las herramientas que nos permitan hacerlo funcionar sin fricciones; o tal vez hay algún elemento que hay que eliminar (como restos incómodos de otros sistemas pasados); o al revés, deberíamos recuperar aspectos (como la religión o ciertos valores) que compensen lo que en el sistema no funciona; o puede que sea un aspecto imposible de eliminar (como la avaricia o la violencia natural humana) lo que eche todo al traste y debamos aceptar lo que tenemos con resignación; o tal vez no es necesario buscar un obstáculo para esto, y pensar que la sociedad está en un continuo proceso de desarrollo y mejora, con unos objetivos trascendentales fijados como la libertad, la igualdad o la justicia, a los cuales nos acercamos con esfuerzo. Lo que, aquí, la reinterpretación de la 
dialéctica por parte de Žižek hace aparecer, es la consideración de que tales fricciones no son por causas externas, sino porque toda estructura social es en sí misma inconsistente e incompleta. Se trata de un cambio de paralaje que nos permite comprender que cualquier realidad no es una totalidad cerrada en su devenir, constituyendo una estructura sin fugas, sino un "todo" incompleto que se sostiene sobre su propia inconsistencia. Nos permite dar cuenta de que las inestabilidades de todo sistema no son defectos de mi comprensión o antagonismos que desestabilizan el sistema haciéndolo progresar, sino el elemento real que lo sostiene. ¿No es esto lo que hace Marx en El capital cuando, analizando la economía política, descomponiéndola en todas sus partes, lo que ilumina son sus contradicciones internas y, además, hace ver cómo son precisamente estas contradicciones internas lo que sostiene el sistema de la economía política en su totalidad?.

Los antagonismos no son el elemento a curar dentro de una sociedad dada, como pensaban los materialistas dialécticos ortodoxos, imaginando un punto de llegada donde se resuelvan las contradicciones, sino que son el punto donde una estructura socio-simbólica se sostiene a sí misma. Por eso la noción vulgar del materialismo, al final se convierte en una herramienta desproblematizadora, pues equivoca dónde está la posibilidad de la acción política y no comprende cómo precisamente el momento de mayor confusión puede ser también aquel en el que el sistema encuentra más oportunidades de autoreproducirse. De hecho, para Žižek, es la categoría de conflicto, de inconsistencia, de falla, de imposibilidad de cierre, el elemento central de la ontología desarrollada a partir del concepto lacaniano de Lo Real, lo que permite pensar, a la vez, tanto la acción política como su imposibilidad. Por eso una totalidad (como el Absoluto hegeliano) "no es una entidad positiva que persiste en su identidad impermeable más allá del mundo transitorio de las cosas finitas" (Žižek, 2015), sino más bien el proceso de surgimiento y caída de las cosas particulares, "el constante proceso de caer", pues "No hay ninguna Sustancia que caiga, se curve, interrumpa el flujo, etc.; la sustancia simplemente es la capacidad infinitamente productiva de tales caídas/cortes/interrupciones, estos son su única realidad" (Žižek, 2015). Y el saber absoluto no es un conocimiento de algo, sino un cambio de paralaje por el cual adoptamos la posición de que saber del objeto es un saber también del sujeto. Que no se trata de un sujeto que mira al mundo como si éste se autopresentase en la forma de un ahí afuera, sino más bien de un sujeto que se sabe a sí mismo como ese ser-ahí incluido en la escena que. Félix Duque escribe que es un saber que consiste en la "comprensión positiva y unitaria de la interna destrucción dialéctica de todo conocimiento que se cree válido por separado, como si fuera justamente algo absoluto" (Duque, 1998). Se trata, por tanto, del punto de vista desde el que se toma el conocimiento, cualquier posición subjetiva, como un saber relativo a un Todo, evitando la tentación de absolutizar dicha situación. El desarrollo dialéctico de la Fenomenología es, de este modo, un proceso de destrucción de toda pretensión de establecer una verdad que sea meramente objetiva. En lugar de esto, Žižek nos dice que tal saber consiste en el momento que la conciencia asume reflexivamente el "fracaso como un resultado positivo, convirtiendo el problema en su propia solución”. (Žižek, 2015)

\section{4. ¿Cómo ser un materialista dialéctico hoy en día?}

Creemos que la aportación que hace la reformulación del materialismo dialéctico por parte de Žižek es innegable como herramienta para el análisis de todo tipo de cuestiones. Lo que no queda tan claro es si la pregunta que se hace Adam Kotsko "¿qué política debería defender hoy un materialista dialéctico?” tiene una respuesta tan evidente. La misma obra de Žižek es titubeante al respecto y da la impresión que oscila entre la defensa de una política revolucionaria activa de acuerdo con el viejo mantra comunista "cuanto peor mejor" y posiciones más sosegadas en las que se acepta provisionalmente la democracia liberal con el propósito de transformarla profundamente. Aquí queremos plantear estos dos extremos y analizar qué pueden tener en común a través de dos de las ideas que Žižek ha desarrollado en su apuesta política: el concepto de revolución como "autogolpe" por un lado, y la posición negativa expresada en el famoso lema de "Bartleby" de Melville que Žižek hace suyo: "preferiría no hacerlo" (Žižek, 2006), por el otro.

Como Žižek ha señalado muchas veces, contrariamente a lo que pueda parecer, la verdadera subsunción del sujeto a las estructuras sociales de dominación no se da en el nivel del acatamiento de las normas, sino precisamente en el de la desidentificación respecto de ellas. La completa captura del sujeto por parte del capitalismo tiene lugar no en el hecho de que este se identifique plenamente con su inscripción simbólica, su posición dentro del sistema, del que no desea apartarse, sino todo lo contrario, en las islas de autoidentidad en las que se ve como independiente y es capaz incluso de transgredir el nivel de las normas sociales. Si esto es así, el escenario pinta mal para las políticas emancipadoras desde el punto de vista de Žižek, puesto que cuanta más ruptura con el sistema se pide, más sujeto se está. Žižek desarrolla esta idea a partir 
del concepto de ideología en Althusser, para el cual la subsunción del sujeto a las estructuras sociales no está en el nivel del pensamiento, sino de las prácticas sociales: "La paradoja fundamental de la relación entre el poder público y lo inevitable de su transgresión es que el sujeto está realmente 'en' (atrapado en la trama del) el poder solo y precisamente en la medida en que no se identifica plenamente con él y mantiene una suerte distancia hacia él" (Žižek, 2002). "Para liberarse efectivamente del yugo de la realidad social existente, es preciso primero renunciar al suplemento transgresivo fantasmático que nos liga a ella" (Žižek, 2002), es decir, lo que podemos llamar con Marx fetichismo.

La cuestión es ¿cómo renunciar a este suplemento?, ¿cómo adoptar una verdadera posición crítica? El problema no es que haya un Otro malvado que nos impone su dominio externamente, sino que nosotros mismos y nuestra propia identidad funciona como vehículo de tal dominación. Por eso, "rechazar radicalmente el sistema incluye rechazar los dispositivos por los cuales nos alejamos de él, convenciéndonos de que no somos 'parte del problema"” (Kotsko, 2008). El golpe que debemos asestar al sistema si realmente queremos hacer un posicionamiento crítico no es contra la ley, las estructuras políticas y sociales, sino contra nosotros mismos. Žižek redefine de este modo lo que podríamos llamar violencia política revolucionaria, aquella que es capaz de romper con la seducción fetichista del sistema. La violencia política debe concebirse como la reforma completa de la sustancia misma del ser del sujeto, de la propia identidad.

Esta idea de autogolpe hay que ponerla en relación directa (dialéctica) con el «preferiría no hacerlo» de Barltleby que Žižek nos propone en Visión de paralaje. ¿En qué sentido puede haber una correspondencia entre el gris oficinista Bartleby que ante la demanda de su jefe responde un "preferiría no hacerlo" y el personaje que interpreta Edward Norton en El club de la lucha (Žižek, 2004) que, frente al despido por parte de su jefe, éste se propina a sí mismo una paliza? Aparentemente la relación entre uno y otro parecen mantener sólo una similitud: la perplejidad del jefe que queda completamente paralizado, incapaz de responder a ninguna de las dos acciones. Sin embargo en todo lo demás parecen posiciones contrarias: en el caso de Bartleby se trata de un empleado que decide no atender la demanda del Otro, mientras que el personaje de El Club de la lucha directamente encarna la propia posición de insignificancia que representa un simple oficinista maltratado por el sistema para el Otro.

Bartleby no es para Žižek como sí lo es para Hardt y Negri (2005), un símbolo de la resistencia pasiva contra las imposiciones del poder mediante el rechazo al trabajo. Esta resistencia, como hemos visto, no puede evitar la trampa del deseo parasitando aquello que pretende negar. De hecho Žižek subraya que la resistencia de Bartleby no es a obedecer las normas, a cumplir con sus obligaciones simbólicas dado que aún cuando se niega educadamente a cumplir con las demandas directas de su jefe, sigue ocupando su lugar como escribiente (Žižek, 2006). De lo que se trata, por tanto, es de romper la seducción fetichista que nos incluye dentro del sistema con todo tipo de prácticas, tanto las que nos unen directamente al capitalismo, como aquellas en que encontramos una identidad a salvo que nos proporcionan una versión de nosotros mismos libre de toda ideología (ecológicas, solidarias, multiculturalistas, respetuosas de las identidades, etc.). Žižek busca una posición subjetiva en la que los individuos acepten sus obligaciones simbólicas pero no se sientan interpelados por ellas.

Por eso es importante leer en paralelo la idea Zizekiana de autogolpe, con la aparente resistencia pasiva de Bartleby, pues nos permite comprender, a mi juicio, qué entiende Žižek aquí por la posición de un verdadero materialista dialéctico. Bartleby acepta su identidad simbólica pero no se siente obligado por ella, no está enganchado con la seducción fetichista de la ley. Esta forma de rechazo tiene la estructura de la figura hegeliana de la "negación de la negación" aunque reformulada al modo zizekiano: inicialmente uno se asume como parte del sistema, acepta la posición que ocupa (la plena aceptación del empleado del El Club de la lucha, como un insignificante ser de maltrato). En un segundo momento se niega esta posición por no alcanzar su propósito (el rechazo de Hart y Negri de incluirse en el sistema). Finalmente se niega esta negación y se recupera la primera posición, el ser parte del sistema pero asumiendo que no alcanza su propósito, es decir, adoptándola sin su suplemento fetichista. Este mismo movimiento coincide con la idea de autogolpe, pues lo que trata de articular Žižek es precisamente la idea de que la plena asunción de la propia identidad simbólica, sin refugiarse en distancias sanadoras, equivale a la propia autodestitución, a la transformación subjetiva que, para Žižek, permitiría abrir el campo de la transformación política. Visto así, podemos afirmar con Rosalind Coward y John Ellis que el sujeto lacaniano es "el nuevo sujeto del materialismo dialéctico" (Coward-Ellis, 1997) ya que se trata de un sujeto que aunque es construido en el seno de procesos sociales y como un efecto de éstos, no puede ser meramente reducido a sus estructuras, pues es precisamente el intento de hacer funcionar sin fricciones un sistema lo que lo desestabiliza 
inevitablemente. Ser materialistas dialécticos hoy en día, por tanto, creemos, desde esta lectura de la obra de Žižek, equivale a tomarse en serio la propia identidad simbólica que nos hace ser parte esencial de la reproducción del sistema respecto del cual queremos adoptar una posición crítica.

\section{Bibliografía empleada.}

BenNETT, J. (2010). Vibrant matter. A political ecology of things. Durham and London: Duke University Press.

CAStro GomeZ, S. (2015). Revoluciones sin sujeto. México: Akal.

CorduA, C. (2013). "Hegel Hoy según Žižek”, en Revista de filosofía, vol.69.

COWARD, R. Y ELLIS, J. (1977). Language and Materialism, Londres: Routledge.

DuQUe, F. (1998). Historia de la filosofía moderna. La era de la crítica. Madrid: Akal, 1998.

HamzA, A. (2016). “Going to One’s Ground: Žižek’s Dialectical Materialism”, en Agon Hamza y Frank Ruda eds. Slavoj Zizek and Dialectical Materialism. Palgrave Macmillan US. Edición de Kindle.

HARDT, M. Y NEGRI. T. (2005). Imperio, Barcelona: Paidós.

Kotsкo, A. (2008). Zizek and Theology. Nueva York: T\&T Clark.

LACAN, J. (2007). El seminario. Libro VII. La ética del psicoanálisis. Buenos Aires: Paidós.

ŽIžEK, S. (2001). El espinoso sujeto. Buenos Aires: Paidós.

ŽıžEK, S. (2002). El frágil absoluto. Valencia: Pre-Textos.

ŽIŽEK, S. (2004). Repetir Lenin. Madrid: Akal.

ŽIŽEK, S. (2006). Arriesgar lo imposible. Conversaciones con Glyn Daly, Madrid: Trotta.

ŽıžEK, S. (2006). Visión De Paralaje. Buenos Aires: Fondo de cultura económica.

ŽIŽEK, S. (2010). El sublime objeto de la ideología. Madrid: Siglo XXI.

ŽIŽEK, S. (2015). Menos que nada. Madrid: Akal 2015.

ŽıžEK, S. (2016). Contragolpe absoluto, Madrid: Akal. 